those derived from pregn-5-enэ, those derived from conanine and those of unknown structure-and then creating further sub-divisions with each alkaloid in turn being discussed in terms of its source, its physical constants, the key experimental evidence leading up to the structural assignment with copious cross-references to established analogies, and finally, its synthesis.

The third chapter, which discusses the conversion of various alkaloids into steroid hormones and other therapeutic agents, should serve to stimulate new interest in a number of these alkaloids as alternative potential raw materials for the commercial preparation of steroidal medicaments.

Chapter 4, dealing with the biological properties and pharmacology of the alkaloids, is again very thorough and, as well as providing a review of the pharmacological literature concerned with the purified principles, includes references to Galenicals obtained from plants of the Apocynaceae since shown to contain steroidal alkaloids.

The work includes both subject and author indexes with 385 references. This book should prove to be of great value both to workers whose special interests lie in the domain of alkaloids and to workers whose special interests lie in the steroid field.

M. Martin-Smith

\section{ORGANIC ANALYSIS}

\section{Organic Functional Group Analysis by Micro and} Semimicro Methods

By N. D. Cheronis and T. S. Ma. Pp. xxv + 696. (New York, London and Sydney : John Wiley and Sons, Inc., 1964.) 188s.

DROFS. Cheronis and Ma, in compiling Organic Functional Group Analysis by Micro and Semimicro Methods, have made available the fruits of their many years experience in the teaching of organic analysis at the City University of New York. The book is described as being intended for students pursuing an advanced course in organic analysis and for organic research chemists who, although they may not wish to analyse their products themselves (and which of them does?), may wish to be familiar with the methods used by those expected to do so. This, according to Prof. Ma, is in order that they might be better able to interpret the results of analysis. No doubt all analysts will sympathize with this objective, but they may also wonder whether Prof. Ma is being a little optimistic. In fact the book will be of value to all practising analysts and not merely to students. The discussions of methods and principles are wide-ranging, the references to original literature copious and the necessary information drawn from them without the inclusion of unnecessary detail.

The authors have divided their book into three parts, dealing with general analytical techniques and methods, analytical methods for particular functional groups, and finally experimental procedures. The general methods section deals with such elementary analytical techniques as weighing, measurement of volume, filtration heating, etc., with special emphasis on micro- and semimicrotechniques. Clearly this part of the book is directed primarily at the student readership. The one criticism of this section is levelled at the chapter dealing with the influence of molecular structure on reaction rates. In an analytical work such as this, discussion of the subject cannot be very detailed, but if it is not it is unlikely to be of much value. Only eight pages are devoted to it, and this amounts to little more than an elaborate statement that reactions are influenced by molecular structure.

Part 2, the critical survey of analytical methods, is a mine of useful information. Functional groups are considered under the headings: oxygen, nitrogen, sulphur, unsaturated and miscellaneous functions. The last of these includes hydrocarbon, organophosphorus, organomercury, organoboron, organosilicon, arsenic and halogen functions and the determination of water. Altogether nearly one hundred functions and methods for their analysis are discussed. Instrumental methods of analysis are not discussed in the first part of the book, but in this section many examples of their use are woven into the text in a balanced manner. It is indeed refreshing to find authors who regard these methods simply as weapons in the analyst's armoury neither to be shunned through ignorance of their potentialities nor enshrined for the same reason.

The final section of the book deals with experimental procedures and is divided into two parts dealing with methods requiring normal and special apparatus. This section, which lists detailed analytical procedures of fiftytwo experiments, no doubt incorporates much of the work and the results of the authors' twelve-year research project on functional group analysis, referred to in the preface. Full details are given for these experiments and the lay-out follows the standard pattern.

Altogether this is a very useful book which, in addition to providing the basis for a course in organic analysis, will provide a great deal of information for organic analysts at all levels.

P. J. COOPER

\section{RADIATION CHEMISTRY IN U.S.S.R.}

\section{Radiolysis of Hydrocarbons}

Edited by A. V. Topchiev. English edition edited by R. A. Holroyd. Pp. xii +232 . (Amsterdam, London and New York: Elsevier Publishing Company, 1964.) $65 s$.

7 HIS English edition of a book edited by the late Academician A. V. Topchiev, who was director of the Petroleum Institute, U.S.S.R. Academy of Sciences, Moscow, is a necessary addition to the library of all radiation chemists. It is one of the few reviews of radiation chemistry in the U.S.S.R. in English. This monograph contains basically a description of the research activities of the Radiation Research Laboratories of the Institute for Petrochemical Synthesis between 1957 and 1961, and it mirrors the scientific interests of these Laboratories.

This book is mainly of interest to those working in the field of radiation chemistry of organic compounds, but it contains interesting chapters dealing with a theory of radiationless energy-transfer and theoretical aspects of hydrocarbon radiolysis which are worth the consideration of all radiation chemists. The latter chapter contains a section on the kinetics of reactions occurring in tracks which requires a sound grasp of statistical methods; a section on the kinetics of radiolysis in the presence of 'hot' atoms and molecular fragments which considers spaceenergy distributions using the method of energy groups frequently used in the theory of neutron deceleration and thermalization; and finally a section dealing with the statistical theory of activated processes initiated by ionizing radiation in condensed systems. This problem is tackled from the point of view of probability theory using the ideas of the statistical theory of oscillations.

The remaining chapters are of a more experimental nature, although attempts are made to connect theory with practice at all times. These chapters contain a wealth of useful and interesting results. Full descriptions of experimental and analytical techniques are given. The headings of these chapters are: "Physicochemical Characteristics of Hydrocarbon Radiolysis"; "Kinetics of Radiolysis of Hydrocarbons"; "Free Radicals in the Radiolysis of Hydrocarbons"; "Radiolysis of Alkanes Adsorbed on Oxide Catalysts"; "Radiation-Thermal Cracking of Hydrocarbons".

Thirteen persons participated in the writing of this monograph and this is apparent to some extent. However, because of the nature of this book this is not a serious fault. What is more annoying is a number of obvious 